\title{
Higgs Mechanism and New Propagator of Massive Vector Bosons
}

\author{
Takehisa Fujita, Naohiro Kanda, Kazuhiro Tsuda \\ Department of Physics, Faculty of Science and Technology, Nihon University, Tokyo, Japan \\ Email: \{fffujita, nkanda, nobita\}@phys.cst.nihon-u.ac.jp \\ Received April 7, 2012; revised May 5, 2012; accepted May 31, 2012
}

\begin{abstract}
We discuss a serious problem related to the Higgs mechanism and show that the unitary gauge which imposes the condition of $\phi=\phi^{\dagger}$ on the Higgs fields does not correspond to a proper gauge fixing. Instead, this is simply a procedure for producing the massive vector boson fields by hand. This suggests that the Lagrangian density of the weak interactions should be reconsidered by starting from the massive vector boson fields which couple to the fermion currents as the initial ingredients. Here, it is shown that the new renormalization scheme with massive vector bosons has no intrinsic problem and the massive vector boson fields do not give rise to any divergences for the physical observables in the renormalization scheme.
\end{abstract}

Keywords: Higgs Mechanism

\section{Introduction}

The whole idea of the symmetry breaking has been critically examined in the recent textbook [1], and the physics of the spontaneous symmetry breaking is, by now, well understood in terms of the standard knowledge of quantum field theory. In particular, if one wishes to understand the vacuum state in a field theory model of fermions, then one has to understand the structure of the negative energy states of the corresponding field theory model. However, it is normally very difficult to construct the vacuum state of the interacting system, and in fact, the exact solution of the model field theory is practically impossible in four dimensions. Nevertheless, the physics of the spontaneous symmetry breaking is now clearly understood and some of the model field theory prefer the vacuum state which breaks the chiral symmetry though there exists no massless (Goldstone) boson.

The most important point of the spontaneous symmetry breaking physics is that the symmetry breaking is only related to the property of the vacuum state. The Lagrangian density should keep the chiral symmetry all the time, but the state may violate the symmetry if the state with broken symmetry is lower than the state that preserves the symmtry.

In this respect, one cannot discuss its physics by rewriting the Lagrangian density into a new shape. As one knows, the property of the vacuum state should be determined from the eigenstate of the total Hamiltonian in the corresponding field theory model. Therefore, the physics of the Higgs mechanism [2] should be reexamined properly since it is related to the spontaneous symmetry breaking physics.

As we will show below, the whole procedure of the Higgs mechanism cannot be justified at all. This is mainly connected to the misunderstanding of the gauge fixing where one degree of freedom of the gauge fields must be reduced in order to solve the equations of motion of the gauge fields. Therefore, one cannot insert the condition of the gauge fixing into the Lagrangian density. This is clear since the Lagrangian density only plays a role for producing the equation of motions. Indeed, the Lagrangian density itself is not directly a physical observable, and the Hamiltonian constructed from the Lagrangian density is most important after the fields are quantized. For the field quantization, one has to make use of the gauge fixing condition which can determine the gauge field $A_{\mu}$ together with the equation of motions. This means that only the final Lagrangian density is relevant to the description of physical observables, and thus the success of the Glashow-Weinberg-Salem model [3-5] is entirely due to the final version of the weak Hamiltonian which is not at all the gauge field theory but is a model field theory of the massive vector fields which couple to the fermion currents.

In this respect, it is very important to examine the renormalizability of the final version of the weak Hamiltonian. Here, we show that the renormalizability of the model field theory can be indeed justified. This is 
basically due to the fact that there is no divergence in the vertex corrections of fermions due to the massive vector boson propagations once we employ a proper propagator of the massive vector bosons. Here, we briefly review how we can obtain the new propagator of the massive vector boson, and the correct shape of the propagator of the massive vector bosons should be given as [6]

$$
D^{\mu v}(k)=-\frac{g^{\mu v}-\frac{k^{\mu} k^{v}}{k^{2}}}{k^{2}-M^{2}+i \varepsilon} .
$$

This shape is determined by solving the equations of motion for the massive vector bosons. As long as we employ the above propagator, we find that the anomalous magnetic moment of electron due to weak $Z^{0}$ bosons does not have any divergences and it is indeed very small number which is consistent with experiment. Thus, one can see that the physical observables with the massive vector boson propagations are all finite and that there are niether conceptual nor technical problems in the renormalization scheme of the massive vector bosons interacting with fermions.

\section{Gauge Fixing}

Now we discuss the basic problem of the Higgs mechanism. The Lagrangian density of the Higgs mechanism is given as

$$
\mathcal{L}=\frac{1}{2}\left(D_{\mu} \phi\right)^{\dagger}\left(D^{\mu} \phi\right)-\frac{1}{4} u_{0}\left(|\phi|^{2}-\lambda^{2}\right)^{2}-\frac{1}{4} F_{\mu \nu} F^{\mu \nu}
$$

where

$$
D_{\mu}=\partial_{\mu}+i g A_{\mu}, \quad F_{\mu v}=\partial_{\mu} A_{-}-\partial_{\nu} A_{\mu} .
$$

Here, we only consider the $U(1)$ case since it is sufficient for the present discussions. The above Lagrangian density is indeed gauge invariant, and in this respect, the scalar field may interact with gauge fields in Equation (2.1). However, it should be noted that there is no experimental indication that the fundamental scalar field can interact with any gauge fields in terms of the Lagrangian density of Equation (2.1).

The equations of motion for the scalar field $\phi$ become

$$
\begin{gathered}
\partial_{\mu}\left(\partial^{\mu}+i g A^{\mu}\right) \phi=-u_{0} \phi\left(|\phi|^{2}-\lambda^{2}\right)-i g A_{\mu}\left(\partial^{\mu}+i g A^{\mu}\right) \phi \\
\partial_{\mu}\left(\partial^{\mu}-i g A^{\mu}\right) \phi^{\dagger}=-u_{0} \phi^{\dagger}\left(|\phi|^{2}-\lambda^{2}\right)+i g A_{\mu}\left(\partial^{\mu}-i g A^{\mu}\right) \phi^{\dagger}
\end{gathered}
$$

On the other hand, the equation of motion for the gauge field $A_{\mu}$ can be written as

$$
\partial_{\mu} F^{\mu v}=g J^{v}
$$

where

$$
J_{\mu}=\frac{1}{2} i\left\{\phi^{\dagger}\left(\partial_{\mu}+i g A_{\mu}\right) \phi-\phi\left(\partial_{\mu}-i g A_{\mu}\right) \phi^{\dagger}\right\} .
$$

One can also check that the current $J_{\mu}$ is conserved, that is

$$
\partial_{\mu} J^{\mu}=0
$$

This Lagrangian density of Equation (2.1) has been employed for the discussion of the Higgs mechanism.

\subsection{Gauge Freedom and Number of Independent Equations}

Now, we should count the number of the degrees of freedom and the number of equations. For the scalar field, we have two independent functions $\phi$ and $\phi^{\dagger}$. Concerning the gauge fields $A_{\mu}$, we have four since there are $A_{0}, A_{1}, A_{2}, A_{3}$ fields. Thus, the number of the independent fields is six. On the other hand, the number of equation is five since the equation for the scalar fields is two and the number of the gauge fields is three. This three can be easily understood, even though it looks that the independent number of equations in Equation (2.5) is four, but due to the current conservation the number of the independent equations becomes three. This means that the number of the independent functions is six while the number of equations is five, and they are not equal. This is the gauge freedom, and therefore in order to solve the equations of motion, one has to put an additional condition for the gauge field $A_{\mu}$ like the Coulomb gauge which means $\nabla \cdot \mathbf{A}=0$. In this respect, the gauge fixing is simply to reduce the redundant functional variable of the gauge field $A_{\mu}$ to solve the equations of motion, and nothing more than that.

\subsection{Unitary Gauge Fixing}

In the Higgs mechanism, the central role is played by the gauge fixing of the unitary gauge. The unitary gauge means that one takes

$$
\phi=\phi^{\dagger}
$$

This is the constraint on the scalar field $\phi$ even though there is no gauge freedom in this respect. For the scalar field, the phase can be changed, but this does not mean that one can erase one degree of freedom. One should transform the scalar field in the gauge transformation as

$$
\phi^{\prime}=e^{-i g \chi} \phi
$$

but one must keep the number of degree of freedom after the gauge transformation. Whatever one fixes the gauge $\chi$, one cannot change the shape of the scalar field $\phi$ since it is a functional variable and must be determined from the equations of motion. The gauge freedom is, of 
course, found in the vector potential $A_{\mu}$ as we discussed above. In this sense, one sees that the unitary gauge fixing is a simple mistake. The basic reason why people overlooked this simple-minded mistake must be due to their obscure presentation of the Higgs mechanism. Also, it should be related to the fact that, at the time of presenting the Higgs mechanism, the spontaneous symmetry breaking physics was not understood properly since the vacuum of the corresponding field theory was far beyond the proper understanding. Indeed, the Goldstone boson after the spontaneous symmetry breaking was taken to be almost a mysterious object since there was no experiment which suggests any existence of the Goldstone boson. Instead, a wrong theory prevailed among physicists. Therefore, they could assume a very unphysical procedure of the Higgs mechanism and people pretended that they could understand it all.

\subsection{Final Lagrangian Density}

After an improper gauge fixing, one arrives at the final Lagrangian density

$$
\begin{aligned}
\mathcal{L}= & \frac{1}{2}\left(\partial_{\mu} \eta\right)\left(\partial^{\mu} \eta\right)-\frac{1}{4} u_{0}\left(|\lambda+\eta(x)|^{2}-\lambda^{2}\right)^{2} \\
& +\frac{1}{2} g^{2}(\lambda+\eta(x))^{2} A_{\mu} A^{\mu}-\frac{1}{4} F_{\mu \nu} F^{\mu v}
\end{aligned}
$$

where we rewrite the Higgs field as

$$
\phi=\phi^{\dagger}=\lambda+\eta(x) \text {. }
$$

Since the real scalar field $\eta$ is supposed to be small and besides a real scalar field is unphysical $[7,8]$, it may be set to zero, that is, $\eta=0$. In this case, we arrive at the following Lagrangian density

$$
L=\frac{1}{2} g^{2} \lambda^{2} A_{\mu} A^{\mu}-\frac{1}{4} F_{\mu \nu} F^{\mu v} .
$$

This should be the final Lagrangian density of the Higgs theory, and it is nothing but the massive vector boson field which has nothing to do with the gauge theory.

\subsection{Proper Gauge Fixing}

For the equations of motion Equations (2.3)-(5), one can make a proper gauge fixing such as the Coulomb gauge $(\nabla \cdot \mathbf{A}=0)$ or the temporal gauge $\left(A_{0}=0\right)$. In this case, one can solve the equations of motion properly, and one can calculate any physical observables which are, in this case, gauge invariant quantities like $F^{\mu v}$ in the classical field theory. Further, we can quantize the fields $\phi$ and $A^{\mu}$ after the gauge fixing, and we can carry out the perturbation theory since we have now the quantized Hamiltonian. But this must be completely different from the Higgs mechanism.

\subsection{Higgs Potential}

In the Lagrangian density of the Higgs mechanism, one assumes a self-interacting field potential

$$
U(\phi)=\frac{1}{4} u_{0}\left(|\phi|^{2}-\lambda^{2}\right)^{2} .
$$

This was originally introduced in the discussion of the spontaneous symmetry breaking physics as a toy model [9]. But in the mean time, this scalar field potential is considered to be a fundamental potential. However, one may ask a question as to where this potential $U(\phi)$ is produced from since the scalar field is obviously not a free field. Unless one can understand the basic origin of this potential $U(\phi)$, it is extremely difficult to accept the Higgs mechanism itself from the fundamental physics point of view.

\section{Renormalization Scheme of Massive Vector Fields}

In 1970's, people found that some experiments indicate there might be heavy vector bosons exchanged between leptons and baryons in the weak processes. Therefore, people wanted to start from the massive vector bosons. However, it was somehow believed among educated physicists that only gauge field theories must be renormalizable. We do not know where this belief came from. In fact, there is no strong reason that only the gauge field theory is renormalizable. It is clear that QED is a gauge theory which is renormalizable, and there is no conceptual problem in QED. At the present stage, one can say that QED can describe most of the experiments related to the electromagnetic processes, except that the Lamb shift treatment is not satisfactory [6]. However, this does not mean that other non-gauge field theory models are not renormalizable. In fact, the basic condition of the renormalizability must be concerned with the coupling constant $g$ which must be dimensionless [1].

\subsection{Renormalizability of Non-Abelian Gauge Field}

However, one should be careful for the renormalizability of the non-abelian gauge field theory. As one can easily convince oneself, the non-abelian gauge theory has an intrinsic problem of the perturbation theory [10]. This is connected to the fact that the color charge in the nonabelian gauge field depends on the gauge transformation, and therefore it cannot be physical observables. This means that the free gauge field which has a color charge is gauge dependent, and thus one cannot develop the perturbation theory in a normal way. In QCD, this is exhibited as the experimental fact that both free quarks and free gluons are not observed in nature. The absence 
of free fields is a kinematical constraint and thus it is beyond any dynamics. Therefore, one cannot discuss the renormalizability of the non-abelian gauge field theory models due to the lack of the perturbation scheme in this model field theory $[1,10]$.

\subsection{Massive Vector Field Theory}

Even though the Higgs mechanism itself has an intrinsic problem, the final Lagrangian density may well be physically interesting. This is clear since, from this Lagrangian density, one can construct the Hamiltonian which can describe the experimental observables. In this respect, we may write the simplest Lagrangian density for two flavor leptons which couple to the SU(2) vector fields $W_{\mu}^{a}$

$$
\begin{aligned}
L= & \bar{\Psi}\left(i \partial_{\mu} \gamma^{\mu}+m\right) \Psi-g J_{\mu}^{a} W^{\mu, a} \\
& +\frac{1}{2} M^{2} W_{\mu}^{a} W^{\mu, a}-\frac{1}{4} G_{\mu \nu}^{a} G^{\mu v, a}
\end{aligned}
$$

where $M$ denotes the mass of the vector boson. The fermion wave function $\Psi$ has two components

$$
\Psi=\left(\begin{array}{l}
\psi_{e} \\
\psi_{v}
\end{array}\right) .
$$

Correspondingly, the mass matrix can be written as

$$
m=\left(\begin{array}{cc}
m_{e} & 0 \\
0 & m_{v}
\end{array}\right) .
$$

The fermion current $J_{\mu}^{a}$ and the field strength $G_{\mu \nu}^{a}$ are defined as

$$
J_{\mu}^{a}=\bar{\Psi} \gamma_{\mu} \tau^{a} \Psi, G_{\mu \nu}^{a}=\partial_{\mu} W_{\nu}^{a}-\partial_{\nu} W_{\mu}^{a} .
$$

This Lagrangian density is almost the same as the standard model Lagrangian density, apart from the Higgs fields.

\subsection{Renormalizability}

Therefore, it should be important to examine whether the renormalization scheme of the massive vector bosons interacting with fermions can be justified or not [11]. In this sense, this simple model of Equation (3.1) is just quite similar to the final Lagrangian density of the standard model itself if we remove some of the degrees of freedom such as the Higgs fields. Here, it is shown below that the massive vector boson does not give rise to any divergences in the vertex corrections of fermions once we employ the proper propagator of the massive vector boson as given in Equation (1.1) [6].

\section{Propagator of Massive Boson}

Here, we briefly review the derivation of the new propagator of the massive vector boson which has recently been evaluated properly in terms of the polarization vector [6]. The correct shape of the boson propagator is found to be the one given as

$$
D^{\mu v}(k)=-\frac{g^{\mu v}-\frac{k^{\mu} k^{v}}{k^{2}}}{k^{2}-M^{2}+i \varepsilon}
$$

This is quite important since this does not generate any quadratic divergences in the self-energy diagrams of fermions any more while the old propagator in the textbooks $D_{\text {old }}^{\mu v}(k)=-\frac{g^{\mu v}-\frac{k^{\mu} k^{v}}{M^{2}}}{k^{2}-M^{2}+i \varepsilon}$ gives rise to the quadratic divergence $[1,12]$.

\subsection{Lorentz Condition of $\boldsymbol{k}_{\mu} \varepsilon^{\mu}=0$}

Here, we briefly explain how we can obtain Equation (4.1). The free Lagrangian density for the vector field $Z^{\mu}$ with its mass $M$ is written as

$$
\mathcal{L}_{Z}=-\frac{1}{4} G_{\mu \nu} G^{\mu \nu}-\frac{1}{2} M^{2} Z_{\mu} Z^{\mu}
$$

with $G^{\mu \nu}=\partial^{\mu} Z^{v}-\partial^{v} Z^{\mu}$. In this case, the equation of motion becomes

$$
\partial_{\mu}\left(\partial^{\mu} Z^{v}-\partial^{v} Z^{\mu}\right)+M^{2} Z^{v}=0 .
$$

Since the free massive vector boson field should have the following shape of the solution

$$
Z^{\mu}(x)=\sum_{\mathbf{k}} \sum_{\lambda=1}^{3}\left(1 / \sqrt{2 V \omega_{\mathbf{k}}}\right) \varepsilon^{\mu}(k, \lambda)\left[c_{\mathbf{k}, \lambda} e^{-i k x}+c_{\mathbf{k}, \lambda}^{\dagger} e^{i k x}\right]
$$

we can insert this solution into Equation (4.2) and obtain the following equation for the polarization vector $\varepsilon^{\mu}$

$$
\left(k^{2}-M^{2}\right) \varepsilon^{\mu}-\left(k_{v} \varepsilon^{v}\right) k^{\mu}=0 .
$$

The condition that there should exist a non-zero solution for the $\varepsilon^{\mu}$ requires that the determinant of the matrix should be zero, namely

$$
\operatorname{det}\left\{\left(k^{2}-M^{2}\right) g^{\mu v}-k^{\mu} k^{v}\right\}=0 .
$$

This equation can be easily solved, and we find the following equation

$$
k^{2}-M^{2}=0
$$

which is the only physical solution of Equation (4.5). Therefore we insert this solution into Equation (4.4) and obtain the equation for the polarization vector $\varepsilon^{\mu}$

$$
k_{\mu} \varepsilon^{\mu}=0
$$

which should always hold. Here, we should note that this process of determining the condition on the wave function of $\varepsilon^{\mu}$ is just the same as solving the free Dirac equation. Obviously this is the most important process of 
determining the wave functions in quantum mechanics, and surprisingly, this has been missing in the treatment of determining not only the massive vector boson propagator but also the photon propagator as well. Also, one can notice that the condition of Equation (4.7) is just the same as the Lorentz gauge fixing condition in quantum electrodynamics (QED), and this is often employed as the gauge fixing. However, one sees by now that the Lorentz condition itself can be obtained from the equation of motion, and therefore it is more fundamental than the gauge fixing, even though the theory of massive bosons has no gauge freedom. This indicates that the Lorentz gauge fixing in QED should not be a proper gauge fixing procedure since the Lorentz gauge fixing cannot give a further constraint on the polarization vector in the perturbation theory of QED. In addition, the number of degrees of freedom for the gauge field can be understood properly since photon must have the two degrees of freedom due to the two constraint equations (the Lorentz condition and the gauge fixing condition).

\subsection{Propagator of Massive Vector Boson}

Now, we can evaluate the propagator of the massive vector field in the S-matrix expression. The second order perturbation of the S-matrix for the bosonic part can be written in terms of the T-product of the boson fields and it becomes

$$
\begin{aligned}
& \left\langle 0\left|T\left\{Z^{\mu}\left(x_{1}\right) Z^{v}\left(x_{2}\right)\right\}\right| 0\right\rangle \\
& =i \sum_{\lambda=1}^{3} \int \frac{\mathrm{d}^{4} k}{(2 \pi)^{4}} \varepsilon^{\mu}(k, \lambda) \varepsilon^{v}(k, \lambda) \frac{e^{i k\left(x_{1}-x_{2}\right)}}{k^{2}-M^{2}+i \varepsilon} .
\end{aligned}
$$

After the summation over the polarization states, we find the following shape for $\sum_{\lambda=1}^{3} \varepsilon^{\mu}(k, \lambda) \varepsilon^{v}(k, \lambda)$ as

$$
\sum_{\lambda=1}^{3} \varepsilon^{\mu}(k, \lambda) \varepsilon^{v}(k, \lambda)=-\left(g^{\mu \nu}-\frac{k^{\mu} k^{v}}{k^{2}}\right)
$$

which satisfies the Lorentz invariance and the condition of the polarization vector $k_{\mu} \varepsilon^{\mu}=0$. One sees that this is the only possible solution. From Equation (4.9), one finds that the right propagator of the massive vector boson should be the one given in Equation (4.1). Here it may be important to note that the polarization vector $\varepsilon^{\mu}(k, \lambda)$ should depend only on the four momentum $k^{\mu}$, and it cannot depend on the boson mass at this expression. Later, one may replace the $k^{2}$ term by $M^{2}$ in case the vector boson is found at the external line. But in the propagator, the replacement of the $k^{2}$ term by $M^{2}$ is not allowed.

\section{Vertex Corrections by Weak Bosons}

Now we can calculate the vertex correction $\Lambda\left(p, p^{\prime}\right)$ of electromagnetic interaction due to the $Z^{0}$ boson. This is a physical process which can be directly related to the physical observables, and therefore we should be concerned with its divergences. The vertex correction $\Lambda\left(p, p^{\prime}\right)$ can be written by evaluating the corresponding Feynman diagrams as [6]

$$
\begin{aligned}
\Lambda^{\rho}\left(p^{\prime}, p\right)= & -i g_{z}^{2} e \int \frac{\mathrm{d}^{4} k}{(2 \pi)^{4}}\left(\frac{g^{\mu \nu}-\frac{k^{\mu} k^{v}}{k^{2}}}{k^{2}-M^{2}+i \varepsilon}\right) \\
& \times \gamma_{\mu} \gamma_{5} \frac{1}{\not p-\not k-m_{e}} \gamma^{\rho} \frac{1}{\not p-\not K-m_{e}} \gamma_{v} \gamma_{5} .
\end{aligned}
$$

\subsection{No Divergences}

First, we show that the apparent logarithmic divergent terms in Equation (5.1) vanish to zero, and this can be easily proved since we can find

$$
\begin{aligned}
\Lambda^{\rho}\left(p^{\prime}, p\right)= & -i e g_{2}^{2} \int \frac{\mathrm{d}^{4} k}{(2 \pi)^{4}} \int_{0}^{1} 2 x \mathrm{~d} x \\
& \times \frac{\left(\gamma_{\mu} k \gamma^{\rho} k \gamma^{\mu}-\frac{k k \gamma^{\rho} k k}{k^{2}}\right)}{\left(k^{2}-s+i \varepsilon\right)^{3}}=0
\end{aligned}
$$

where $s=M^{2}(1-x)+m_{e}^{2} x^{2}$. Therefore, there is no logarithmic divergence for the vertex correction from the weak massive boson propagations. This is very important in that the physical processes do not have any divergences when we make use of the massive boson propagator of Equation (4.1).

\subsection{Electron $g-2$}

Now we should calculate the electron $g-2$ by $Z^{0}$ boson propagation. This is just the finite part of the vertex correction due to the $Z^{0}$ boson, and it is calculated to be

$$
\Lambda^{\rho}\left(p^{\prime}, p\right) \simeq e \gamma^{\rho} \frac{\alpha_{z}}{4 \pi}\left(\frac{m_{e}}{M}\right)^{2} \ln \left(\frac{M}{m_{e}}\right)^{2}
$$

where $\alpha_{z}=g_{z}^{2} 4 \pi \simeq 2.7 \times 10^{-3}$. Therefore, the electron $g-2$ should be modified to

$$
\frac{g-2}{2} \simeq \frac{\alpha_{z}}{4 \pi}\left(\frac{m_{e}}{M}\right)^{2} \ln \left(\frac{M}{m_{e}}\right)^{2} \simeq 2 \times 10^{-13}
$$

which is a very small effect, and it is indeed consistent with $g-2$ experiment of electron. We should note that, if we employed the old but standard propagator of the massive vector boson as given in the textbooks [12], then we would have obtained a very large effect on the electron $g-2$. But still this large contribution should be 
obtained only under the assumption that we would have successfully treated the quadratic and logarithmic divergences in some way or the other by renormalizing them into the fermion self-energy terms which should have the quadratic and logarithmic divergences. In this sense, if one uses the old propagator, then one has to make use of the fermion self-energy while the new propagator enables us to calculate physical observables without making use of the fermion self-energy contributions.

\section{Conclusions}

The intrinsic problem of the Higgs mechanism is discussed in terms of the gauge fixing condition. This is also related to the understanding of the spontaneous symmetry breaking physics. Here, we have shown that the Higgs mechanism cannot be justified since the gauge invariance of the Lagrangian density is violated by hand. However, we believe that the final version of the weak Hamiltonian should be correct, and therefore we have discussed the renormalization scheme of the massive vector bosons. Here, we have shown that the vertex corrections do not have any divergences as long as we employ the proper propagators of massive vector bosons. Therefore we can calculate physical observables without worrying about the renormalization procedures in the weak interaction model Hamiltonian with the massive vector boson fields.

The basic reason why the standard model Hamiltonian becomes a reasonable model is due to the fact that they make mistakes twice and thus it gets back to the right Hamiltonian which can describe the nature. The first mistake is related to the non-abelian character of the gauge field theory model. As one knows, the $\mathrm{SU}(2)$ charge is gauge dependent, and therefore it cannot be a physical observable. Thus, it is impossible to develop the perturbation theory since the unperturbed Hamiltonian is gauge dependent. The second mistake is concerned with the breaking of the local gauge invariance in terms of Higgs mechanism, and it is, of course, an incorrect treatment as we have shown in this paper.

In this respect, as long as we carefully examine the renormalization scheme of the field theory models, we can say that the massive vector fields do not give rise to any difficulties in the renormalization scheme. However, the basic difficulty of the renormalization scheme should be concerned with the massless nature of the gauge field in QED. Indeed, as one knows, the vertex corrections of fermions due to photon propagations contain an infra-red divergence [13], even though people throw it away since it cannot be renormalized into the fermion wave function. This may indicate that the renormalization scheme of QED is still not necessarily complete in some sense since the logarithmic divergence of the Lamb shift energy is not yet understood well.

\section{REFERENCES}

[1] T. Fujita, "Symmetry and Its Breaking in Quantum Field Theory," 2nd Edition, Nova Science Publishers, 2011.

[2] P. W. Higgs, "Broken Symmetries, Massless Particles and Gauge Fields," Physics Letters, Vol. 12, No. 2, 1964, pp. 132-133. doi:10.1016/0031-9163(64)91136-9

[3] S. L. Glashow, "Partial-Symmetries of Weak Interactions," Nuclear Physics, Vol. 22, No. 4, 1961, pp. 579588. doi:10.1016/0029-5582(61)90469-2

[4] S. Weinberg, "A Model of Leptons," Physical Review Letters, Vol. 19, 1967, pp. 1264-1266. doi:10.1103/PhysRevLett.19.1264

[5] A. Salam, "In Elementary Particle Physics (Nobel Symposium No. 8)," Almqvist and Wilsell, Stockholm, 1968.

[6] T. Fujita and N. Kanda, "No Anomaly and New Renormalization Scheme," 2012.

http://www.quant-ph.cst.nihon-u.ac.jp/fffujita/AnomalyR S.pdf

[7] T. Fujita, S. Kanemaki, A. Kusaka and S. Oshima, "Mystery of Real Scalar Klein-Gordon Field," physics/ 0610268.

[8] S. Kanemaki, A. Kusaka, S. Oshima and T. Fujita, "Problems of Scalar Bosons," In: T. Fujita, Ed., New Fundamentals in Fields and Particles, Research Signpost, 2008.

[9] J. Goldstone, "Field Theories with Superconductor Solutions," Nuovo Cimento, Vol. 19, No. 1, 1961, pp. 154-164. doi:10.1007/BF02812722

[10] T. Fujita, "Physical Observables in Gauge Field Theory," In: T. Fujita, Ed., New Fundamentals in Fields and Particles, Research Signpost, 2008.

[11] K. Nishijima, "Fields and Particles," W. A. Benjamin, Inc., New York, 1969.

[12] F. Mandl and G. Shaw, "Quantum Field Theory," John Wiley \& Sons, New York, 1993.

[13] J. D. Bjorken and S. D. Drell, "Relativistic Quantum Mechanics," McGraw-Hill Book Company, New York, 1964. 